\title{
Proporções e Coeficientes de Crescimento dos não-Componentes da Carcaça de Cordeiros e Cordeiras em Diferentes Métodos de Alimentação 1
}

\author{
Gilberto Teixeira da Rosa², Cleber Cassol Pires ${ }^{3}$, José Henrique Souza da Silva ${ }^{4}$, Otacílio \\ Silva da Motta ${ }^{5}$
}

\begin{abstract}
RESUMO - O experimento foi realizado com o objetivo de estudar as proporções dos não-componentes da carcaça e o crescimento relativo dos mesmos. Foram utilizados 45 cordeiros, 22 machos não castrados e 23 fêmeas da raça Texel. Desses, sete foram abatidos no início do experimento 24 horas após o parto, e os demais assim que atingiam os pesos de 25 ou $33 \mathrm{~kg}$. Foi adotado um delineamento inteiramente casualizado com um arranjo fatorial 3 x 2 × 2 (três métodos, dois sexos e dois pesos de abate). Ocorreu diminuição das proporções de cabeça e patas com a elevação do peso de abate, verificando-se valores de 4,44\% e 2,63\% para pesos de $25 \mathrm{~kg}$ e de $3,76 \%$ e 2,43\% para pesos de $33 \mathrm{~kg}$, respectivamente.Entre sexos a maior proporção de gordura perirenal foi obtida para as fêmeas e entre pesos de abate, para $33 \mathrm{~kg}$.As proporções de pele, fígado e coração não diferiram entre métodos de alimentação, sexos e pesos de abate. A maior proporção de rúmen-retículo foi dos cordeiros desmamados aos 45 dias mas, entre sexos e pesos de abate foram semelhantes.O crescimento relativo das patas, coração, rins e pulmão + traquéia é precoce, enquanto o da pele, fígado e gordura perirenal é isométrico, em relação ao peso de corpo vazio. O rúmen + retículo, independentemente de método e sexo, foi de crescimento tardio, cujos coeficientes variaram entre 2,22 e 2,47 .
\end{abstract}

Palavras-chave: coeficientes alométricos, componentes do peso vivo, rendimento, ovinos

\section{Proportions and Coefficients of Growth Components, Non-Carcass of the Live Weight of Male and Female Lambs under Different Feeding Methods}

\begin{abstract}
The experiment was carried out to study the components proportions, non-carcass of the body weight and their relative growth. It was used 45 lambs: 22 non-castrated male and 23 females of Texel breed. From that group, 7 were slaughtered in the beginning of the experiment and the others when they reached 25 or $33 \mathrm{~kg}$. A randomly delineation adopted was by a factorial arrangement of 3:2:2 (three methods, two sexes and two weights of slaughter). The head and paws proportions decreased with elevation of the slaughter weight with values of $4.44 \%$ and $2.63 \%$ to $25 \mathrm{~kg}$; $3.76 \%$ and $2.43 \%$ to $33 \mathrm{~kg}$, respectively. Among sex, the main proportion of the kidney fat was reached $33 \mathrm{~kg}$ to females and among the weights of slaughter. The skin, liver and heart proportions did not distinguished among feeding methods, sexes and weights of slaughter. The large proportion of rumen and small net was for lambs weaned in 45 days, but between sexes and weights of slaughter was similar. The paws, heart, kidney, lung and trachea relative growth is premature, whereas to skin, liver and kidney fat is isometric, in relation to the weight of the empty body. The rumen+small net had premature growth regardless sex and feeding methods with coefficients ranging from 2.22 to 2.47 .
\end{abstract}

Key Words: components live weight, yield, allometric coefficients, sheep

\section{Introdução}

Ao sacrificar um animal, além da carcaça, obtémse certa quantidade de subprodutos, também aproveitáveis, conhecidos como não-componentes da carcaça, em função da sua importância econômica. Os primeiros a utilizarem a denominação de quinto quarto foram os açougueiros franceses, com objetivo de designar por esse quarto uma porção suplementar que poderiam comercializar, além dos outros quatro quartos que se subdivide a carcaça (Peyron, 1963). O quinto quarto serve para amenizar os custos de abate do frigorífico, mas o produtor recebe um preço global pela carcaça, no qual não aparecem os custos de abate, nem o valor do quinto quarto, o que explica o desinteresse pelo mesmo, tanto na Espanha como no Brasil, onde os animais são vendidos a peso vivo (Delfa et al., 1991).

Assim, faz-se cada vez mais necessária a obtenção de informações não só da carcaça, mas também

\footnotetext{
${ }^{1}$ Parte da dissertação de mestrado apresentado à UFSM.

2 Zootecnista, Mestre em Zootecnia na UFSM. E.mail: a9860181@zipmail.com.br

3 Médico Veterinário, Prof. Dr. do Depto de Zootecnia na UFSM. E.mail: cpires@ccr.ufsm.br

4 Eng.-Agrônomo, Prof. Dr. do Depto de Zootecnia na UFSM. E.mail jhen@ccr.ufsm.br

5 Zootecnista, Mestre em Zootecnia na UFSM.
} 
dos demais constituintes do peso vivo cuja comercialização agregará maior valor econômico ao animal como um todo, motivando os produtores a ter maiores cuidados com o rebanho.

Segundo Lyford (1993), o rápido incremento de peso do tecido do intestino delgado em cordeiros cessa entre a $8^{\mathrm{a}}$ e $9^{\mathrm{a}}$ semana de idade, e a ordem de crescimento dos órgãos do aparelho digestivo desde o nascimento até a idade adulta é: rúmen, retículo, omaso, ceco, intestino grosso, reto, abomaso e esôfago. Para o mesmo autor, o crescimento rápido do pré-estômago começa no momento em que o animal inicia a ingestão de alimentos sólidos, e ao mesmo tempo que o crescimento do intestino delgado é interrompido, o pré-estômago passa a ser o órgão dominante a partir da $12^{\mathrm{a}}$ e $16^{\mathrm{a}}$ semana de idade.

Segundo Black (1989), o crescimento de órgãos como fígado, rins e trato digestivo implicam em rápidas mudanças de peso, quando o animal recebe dieta acima da mantença, e apresentam notável atrofia, quando recebem alimentações abaixo do nível de mantença. Drouillard et al. (1991) observaram que os órgãos de importância para o animal, como cérebro, olhos, extremidades, pulmões, rins, coração, esôfago, abomaso e intestino delgado, são proporcionalmente maiores ao nascimento e conseqüentemente, desenvolvem-se menos na vida pós-natal. $\mathrm{O}$ trabalho teve como objetivo de verificar as proporções e o crescimento relativo dos não-componentes da carcaça (quinto quarto).

\section{Material e Métodos}

O experimento foi conduzido no Setor de Ovinocultura do Departamento de Zootecnia da Universidade Federal de Santa Maria (UFSM) do Rio Grande do Sul.

Foram utilizados 45 cordeiros, (22 machos não castrados e 23 fêmeas) da raça Texel. Desses, 3 machos e 4 fêmeas foram abatidos no início do experimento logo após o nascimento (abate inicial). Os demais, 24 horas após o nascimento, foram divididos por sexo e pesos de abate, pesados e distribuídos aleatoriamente com suas respectivas mães, em três métodos de alimentação: M1 - Alimentação com silagem de milho para o conjunto ovelha + cordeiro e fornecimento de concentrado apenas aos cordeiros na forma de creep feeding do nascimento ao desmame, aos 60 dias de idade e, após, fornecimento de silagem + concentrado para os cordeiros até o abate; M2-
Alimentação com silagem de milho para o conjunto ovelha + cordeiro e fornecimento de concentrado apenas aos cordeiros na forma de creep feeding do nascimento ao desmame, aos 45 dias de idade e após fornecimento de silagem + concentrado para os cordeiros até o abate; M3 - Alimentação com silagem de milho + concentrado para o conjunto ovelha + cordeiro, do nascimento ao desmame, aos 60 dias de idade e após fornecimento de silagem + concentrado para os cordeiros até o abate.

Foi utilizado um galpão coberto com pé direito de 3 metros, subdividido em três baias, medindo $80 \mathrm{~m}^{2}$ cada uma, providas de comedouro e bebedouro, revestidas com piso de cimento. Em duas dessas, foi preparado um local de forma, que somente os cordeiros tivessem acesso ao concentrado (Creep Feeding).

Todos os cordeiros e cordeiras eram de parto simples, sendo o M2 e M3 constituídos, cada um, por 13 cordeiros ( 7 machos e 6 fêmeas) e o M1, por 6 machos e 6 fêmeas. Em cada método e para cada sexo, os pesos de abate foram de 25 e $33 \mathrm{~kg}$ de peso vivo.

Durante o período experimental, foram utilizadas três dietas. No M3 foi fornecida uma ração para atender as exigências nutricionais das ovelhas em lactação, composta por silagem de milho + concentrado. O concentrado utilizado era constituído por milho desintegrado, farelo de soja, calcário calcítico, fosfato bicálcico e cloreto de sódio, com uma proporção de volumoso : concentrado de 70:30\% na matéria seca (MS). Para o M1 e M2 foi fornecido apenas silagem de milho para o conjunto ovelha + cordeiro e no creep feeding, foi fornecido para os cordeiros até o desmame apenas concentrado, constituídos pelos ingredientes acima citados e ofertados numa quantidade equivalente a $2 \%$ do peso vivo médio do lote de cada método, sendo a suplementação mineral fornecida em cocho separado. Do desmame ao abate, foi formulada uma dieta com silagem de milho + concentrado (milho desintegrado, farelo de soja, calcário calcítico, fosfato bicálcico e cloreto de sódio), com uma proporção volumoso : concentrado de 50:50\% na matéria seca (MS). Todas as dietas utilizadas foram calculadas de acordo com o NRC (1985), com exceção da dieta oferecida as ovelhas dos métodos 1 e 2 , que receberam somente silagem.

Os animais foram alimentados duas vezes ao dia, sendo os horários de alimentação às 8 e 16 h. A quantidade diária oferecida foi ajustada em função da sobra observada do dia anterior, a qual foi controlada 
para que fosse mantida em torno de $15 \%$ da quantidade oferecida, de modo a garantir o consumo voluntário dos animais.

Os cordeiros foram pesados após jejum de sólido por 14 horas e imediatamente sacrificados. Após o abate de cada animal, foram pesados a pele, patas, cabeça, fígado, pulmão + traquéia, coração, gordura interna, rins, gordura perirenal. O rúmen, omaso, abomaso, intestino delgado e intestino grosso foram pesados cheio e após lavagem e escorrimento de água foram novamente pesados (peso vazio).

A porcentagem de pele, gordura perirenal, cabeça, pulmão + traquéia, coração, patas, rins, fígado, rúmen + retículo, gordura interna e aparelho digestivo (rúmen-retículo, omaso, abomaso, intestino grosso e delgado) foi obtida em relação ao peso de corpo vazio (PCV).

As análises estatísticas foram realizadas utilizando-se o pacote estatístico SAS (1990). Para avaliação do crescimento relativo, utilizou-se a equação exponencial de Huxley (1932), onde $\mathrm{y}=\mathrm{ax}^{\mathrm{b}}$, transformada logaritmicamente em um modelo linear, logy = $\log \mathrm{a}+\mathrm{b} \log \mathrm{x}$, sendo que: $\mathrm{y}=$ peso do coração, pulmão + traquéia, fígado, patas, pele, cabeça, aparelho digestivo, rúmen + retículo, gordura interna, rins, e gordura perirenal. "a" é a intercepção do logaritmo da regressão linear sobre y e "b" é o coeficiente de crescimento relativo ou coeficiente de alometria e " $x$ " para o peso de corpo vazio. Nos diferentes métodos de alimentação e sexo, foram utilizados todos os cordeiros do experimento, incluindo os abatidos ao nascimento, utilizando o seguinte modelo matemático:

$$
\mathrm{Yij}=\mathrm{m}+\mathrm{b}(\mathrm{Xij}-\mathrm{X})+\mathrm{Eij}
$$

em que: Yij = logaritmo de base 10 do peso do órgão, tecido ou partes da carcaça; $\mathrm{m}=$ efeito da média; $\mathrm{Xij}=\log$ aritmo do peso de corpo vazio ou peso de carcaça fria do animal $\mathrm{j}$ do peso de abate $\mathrm{i}$, sendo $\mathrm{J}=$ 1 ....6 e i $=1$.....4; $\mathrm{X}=$ média do logaritmo de peso de corpo vazio ou peso de carcaça fria; $\mathrm{Eij}=$ erro aleatório.

Para verificação da hipótese $b=1$, foi utilizado o teste " $\mathrm{t}$ " de (Student) $(\mathrm{P}<0,05)$.

$\mathrm{Se} b=1, \mathrm{o}$ crescimento foi denominado isogônico, indicando que as taxas de crescimento de " $\mathrm{X}$ " e " $\mathrm{Y}$ " foram semelhantes no intervalo considerado. Quando $b \neq 1$, o crescimento é heterogônico, sendo precoce se $\mathrm{b}<1$, e tardio se $\mathrm{b}>1$.

\section{Resultados e Discussão}

Na Tabela 1, são apresentados os valores percentuais de cabeça, patas, pele, rins, gordura perirenal, fígado, pulmão mais traquéia e coração em relação ao peso do corpo vazio (PVC).

Houve diferença para a proporção de rins do M2 em relação aos demais métodos de alimentação. Entre sexos, a maior proporção foi para os machos e entre pesos de abate a maior proporção dos rins foi dos animais sacrificados com peso mais leve. Para gordura perirenal ocorreu diferença significativa entre M2 e M3, entre sexo e peso de abate. A diferença quanto aos métodos de alimentação está relacionada ao peso ao desmame, quando os animais do $\mathrm{M} 2$ foram mais leves ocasionando que os mesmos fossem também mais magros ao abate, o que trouxe como consequência menor deposição de gordura interna e externa. A maior proporção da gordura perirenal nas fêmeas se explica em função que estas são mais precoces, quanto a deposição de gordura, em relação aos machos. Para Colomer-Rocher (1974) a taxa de desenvolvimento da gordura perirenal é similar à da gordura total da carcaça e pode ser um indicador do estado de engorduramento geral da mesma.

Os resultados encontrados para gordura perirenal nessa pesquisa são concordantes com Oliver et al. (1967) que afirmam haver diferença significativa entre machos e fêmeas, sendo maior nas fêmeas e não apresentando diferença entre machos castrados e inteiros.

As proporções da pele, fígado e coração são semelhantes entre métodos de alimentação, sexo e pesos de abate e as do pulmão + traquéia diferem apenas entre pesos de abate isto é, ao menor peso de abate correspondeu a maior proporção.

Os resultados da Tabela 1 também são concordantes com os encontrados por Huidobro \& Villapadierna (1992), os quais observaram maiores proporções de cabeça e patas para cordeiros abatidos com $25 \mathrm{~kg}$ em relação aos animais abatidos com $35 \mathrm{~kg}$ de peso vivo, sendo os valores encontrados para cabeça de (4,17 e 3,79\%) e patas (3,18 e 2,75\%), respectivamente para 25 e $35 \mathrm{~kg}$, e para coração, fígado e rins não observaram diferença sendo: coração $(0,44$ e $0,44 \%)$, fígado $(2,26$ e $2,11 \%)$ e rins $(0,41$ e $0,37 \%$ ) também para animais abatidos aos 25 e $35 \mathrm{~kg}$, 
Tabela 1 - Proporções médias dos componentes do peso vivo em relação ao peso de corpo vazio nos métodos de alimentação (M1, M2, M3) de machos (M) e fêmeas (F) e pesos de abate de $25 \mathrm{~kg}(\mathrm{P} 1)$ e $33 \mathrm{~kg}$ (P2) de peso vivo

Table 1 - Average proportions of the components of the live weight in relation to the empty body weight in feeding methods (M1, M2, M3) of male (M) and female (F); and weights of slaughter of $25 \mathrm{~kg}(P 1)$ and $33 \mathrm{~kg}(P 2)$ to live body weight

\begin{tabular}{lccccccc}
\hline $\begin{array}{l}\text { Componentes } \\
\text { Components }\end{array}$ & $\mathrm{M} 1$ & $\mathrm{M} 2$ & $\mathrm{M} 3$ & $\mathrm{M}$ & $\mathrm{F}$ & $\mathrm{P} 1$ & $\mathrm{P} 2$ \\
\hline $\begin{array}{l}\text { Cabeça } \\
\text { Head }\end{array}$ & 4,218 & 4,288 & 3,815 & 4,166 & 4,048 & $4,448^{\mathrm{a}}$ & $3,769^{\mathrm{b}}$ \\
$\begin{array}{l}\text { Patas } \\
\text { Paws }\end{array}$ & $2,347^{\mathrm{b}}$ & $2,602^{\mathrm{a}}$ & $2,642^{\mathrm{a}}$ & 2,608 & 2,452 & $2,626^{\mathrm{a}}$ & $2,434^{\mathrm{b}}$ \\
$\begin{array}{l}\text { Pele } \\
\text { Kkin }\end{array}$ & 13,426 & 12,499 & 12,578 & 12,558 & 14,222 & 12,747 & 12,921 \\
$\begin{array}{l}\text { Rins } \\
\text { Kidneys }\end{array}$ & $0,345^{\mathrm{b}}$ & $0,376^{\mathrm{a}}$ & $0,349^{\mathrm{b}}$ & $0,378^{\mathrm{a}}$ & $0,335^{\mathrm{b}}$ & $0,377^{\mathrm{a}}$ & $0,337^{\mathrm{b}}$ \\
$\begin{array}{l}\text { Gordura perirenal } \\
\text { Kidneylikefat }\end{array}$ & $0,806^{\mathrm{ab}}$ & $0,636^{\mathrm{b}}$ & $0,947^{\mathrm{a}}$ & $0,663^{\mathrm{a}}$ & $0,930^{\mathrm{b}}$ & $0,703^{\mathrm{a}}$ & $0,889^{\mathrm{b}}$ \\
$\begin{array}{l}\text { Fígado } \\
\begin{array}{l}\text { Liver } \\
\text { Pulmão+ traquéia }\end{array}\end{array}$ & 1,985 & 2,047 & 1,912 & 1,989 & 1,973 & 2,017 & 1,945 \\
$\begin{array}{l}\text { Lung + trachea } \\
\text { Coração }\end{array}$ & 1,382 & 1,333 & 1,339 & 1,373 & 1,33 & $1,448^{\mathrm{a}}$ & $1,225^{\mathrm{b}}$ \\
\begin{tabular}{l} 
Heart \\
\hline
\end{tabular} & 0,451 & 0,457 & 0,484 & 0,476 & 0,452 & 0,477 & 0,551 \\
\hline
\end{tabular}

Médias seguidas na linha para (M1, M2 e M3) (M e F) (P1 e P2), com letras desiguais são significativamente diferentes pelo teste Pdiff $(\mathrm{P}<0,05)$.

Averages with unequal letters following in line to (M1, M2 and M3) ( $M$ and F) (P1 and P2) are significantly different by Pdiff Test ( $P<.05)$.

respectivamente. Para pulmão, os referidos autores também verificaram diferença $(\mathrm{P}<0,05)$, com animais abatidos aos $25 \mathrm{~kg}$ com $1,74 \%$ e aos $35 \mathrm{~kg}$ com $1,60 \%$.

Jenkins \& Leymaster (1993), ao trabalharem com animais abatidos em intervalos a partir do nascimento até 48 meses de idade, constataram que a proporção dos componentes das partes não aproveitáveis relativa ao peso corporal vazio foi maior ao nascimento e constantemente declinou com a idade, sugerindo que os padrões de desenvolvimento dos componentes do corpo podem ser estabelecidos pela exigência funcional.

Os coeficientes de alometria para patas, pele e cabeça obtidos através da regressão do logaritmo do peso de cada órgão, em função do peso de corpo vazio $(\mathrm{PCV})$, são apresentados na Tabela 2. Os resultados indicam que as patas são de crescimento precoce $(b<1)$; a pele e a cabeça de crescimento intermediários, pois crescem com a mesma velocidade que o todo $(b=1)$. No caso dos machos do $M 1$, a pele e a cabeça foram de crescimento precoce $(b<1)$ e os do M2, apenas a cabeça foi de crescimento precoce. Os coeficientes obtidos para patas são semelhantes aos de Silva et al. (1999), o qual verificou valores de 0,613. Os resultados referentes ao crescimento da cabeça exceto machos do M1 e M2, concordam com os encontrados por Osório \& Guerreiro (1994), que obteve coeficiente de alometria isogônico em relação ao peso vivo. Com relação à pele, Roque et al. (1997) encontraram desenvolvimento tardio, discordando dos encontrados nesta pesquisa, onde observou-se crescimento isogônico $(b=1)$.

Huidobro \& Cañeque (1994) constataram um desenvolvimento precoce para patas e pele, discordando com os coeficientes de crescimento encontrado para pele neste trabalho. Segundo Butterfield (1988), a pele tem um coeficiente isométrico ou ligeiramente negativo, embora existam variações em função da raça.

Os dados apresentados por Roque (1998), para as raças Romney Marsh e Texel, também discordam dos apresentados na Tabela 2, pois, para a cabeça, foram obtidos coeficientes de 0,587 e 0,582 para as respectivas raças. Isso possivelmente deve-se a diferença quanto ao sistema de produção utilizado pelo autor citado e o do presente trabalho.

Os coeficientes de alometria encontrados para coração, pulmão + traquéia e fígado, obtidos através da regressão do logaritmo do peso de cada órgão em função do peso de corpo vazio (PCV), são apresentados na Tabela 3.

Os coeficientes de alometria dos componentes do 
Tabela 2 - Parâmetros (A e b) das equações de regressão do logaritmo do peso das patas, pele e cabeça em função do peso de corpo vazio, erro padrão, teste $t(H o: b=1)$ e coeficientes de determinação para sexo e métodos de alimentação

Table 2 - Parameters ( $A$ and $b)$ of the regression equations of the logarithmic of weight of paws, skin and head in relation to the weight of the empty body, standard error, test $t(H o: b=1)$ and coefficients of determination to sex and feeding methods

\begin{tabular}{|c|c|c|c|c|c|c|c|c|c|}
\hline & \multicolumn{3}{|c|}{$\begin{array}{l}\text { Patas } \\
\text { Paws }\end{array}$} & \multicolumn{3}{|c|}{$\begin{array}{l}\text { Pele } \\
\text { Skin }\end{array}$} & \multicolumn{3}{|c|}{$\begin{array}{c}\text { Cabeça } \\
\text { Head }\end{array}$} \\
\hline & M1 & M2 & M3 & M1 & M2 & M3 & M1 & M2 & M3 \\
\hline \multicolumn{10}{|l|}{ Machos } \\
\hline \multicolumn{10}{|l|}{ Males } \\
\hline A & 0,619 & 0,418 & 0,334 & $-0,862$ & $-0,884$ & $-0,667$ & $-0,093$ & $-0,133$ & 0,754 \\
\hline B & 0,487 & 0,545 & 0,569 & 0,773 & 0,998 & 0,938 & 0,711 & 0,722 & 0,482 \\
\hline $\mathrm{Sd}$ & 0,088 & 0,026 & 0,019 & 0,041 & 0,049 & 0,031 & 0,026 & 0,022 & 0,247 \\
\hline \multicolumn{10}{|l|}{ Teste $\mathrm{t}$} \\
\hline Test $t$ & $\mathrm{~S}$ & $\mathrm{~S}$ & $\mathrm{~S}$ & $\mathrm{~s}$ & $\mathrm{~ns}$ & $\mathrm{~ns}$ & $\mathrm{~S}$ & S & $\mathrm{ns}$ \\
\hline $\mathrm{R}^{2}$ & 81,43 & 98,26 & 99,13 & 98,08 & 98,12 & 99,12 & 99,11 & 99,27 & 32,34 \\
\hline \multicolumn{10}{|l|}{ Fêmeas } \\
\hline \multicolumn{10}{|c|}{ Females } \\
\hline A & 0,636 & 0,503 & 0,482 & $-0,685$ & $-0,526$ & $-0,734$ & $-0,769$ & $-0,778$ & $-0,765$ \\
\hline B & 0,482 & 0,520 & 0,525 & 0,956 & 0,912 & 0,970 & 0,857 & 0,859 & 0,855 \\
\hline $\mathrm{Sd}$ & 0,040 & 0,049 & 0,035 & 0,056 & 0,037 & 0,042 & 0,176 & 0,157 & 0,155 \\
\hline \multicolumn{10}{|l|}{ Teste $\mathrm{t}$} \\
\hline Test $t$ & S & S & $\mathrm{S}$ & $\mathrm{ns}$ & $\mathrm{ns}$ & $\mathrm{ns}$ & $\mathrm{ns}$ & $\mathrm{ns}$ & $\mathrm{ns}$ \\
\hline $\mathrm{R}^{2}$ & 95,41 & 95,76 & 96,52 & 97,66 & 98,71 & 98,54 & 77,19 & 78,96 & 79,13 \\
\hline
\end{tabular}

M1 = método um; M2 = método dois e M3 = método três.

${ }^{*} \mathrm{~A}=$ intercepto; * $\mathrm{b}=$ coeficiente alométrico; * $\mathrm{sd}=$ erro-padrão; $\mathrm{s}=$ significativo para $(\mathrm{P}<0,05)$; ns = não-significativo para $(\mathrm{P}>0,05)$. $M 1=$ method $1 ; M 2=$ method $2 ; M 3=$ method $3 ; A=$ intercept; $b=$ allometric coefficient $; s d=$ standard error; $s=$ significant at $(P<.05) ;$ ns = not significant at $(P>.05)$.

Tabela 3 - Parâmetros das equações de regressão ( $\mathrm{A}$ e b) do logaritmo do peso do coração, pulmão + traquéia e fígado em função do logaritmo do peso de corpo vazio, erro padrão, teste t $(H o: b=1)$ e coeficientes de determinação para sexo e métodos de alimentação

Table 3 - Parameters ( $A$ and $b$ ) of the regression equations of the logarithmic of weight of paws, skin and head in relation to the weight of the empty body, standard error, test $t(H o: b=1)$ and coefficients of determination, according to the sex and feeding methods

\begin{tabular}{|c|c|c|c|c|c|c|c|c|c|}
\hline & \multicolumn{3}{|c|}{$\begin{array}{c}\text { Coração } \\
\text { Heart }\end{array}$} & \multicolumn{3}{|c|}{$\begin{array}{c}\text { Pulmão + Traquéia } \\
\text { Lung+ Trachea }\end{array}$} & \multicolumn{3}{|c|}{$\begin{array}{l}\text { Fígado } \\
\text { Liver }\end{array}$} \\
\hline & M1 & M2 & M3 & M1 & M2 & M3 & M1 & M2 & M3 \\
\hline \multicolumn{10}{|c|}{ Machos } \\
\hline \multicolumn{10}{|l|}{ Males } \\
\hline A & $-0,738$ & $-0,669$ & $-0,899$ & $-0,862$ & $-0,098$ & $-0,990$ & $-1,437$ & $-1,531$ & $-1,347$ \\
\hline $\mathrm{b}$ & 0,636 & 0,617 & 0,681 & 0,773 & 0,567 & 0,806 & 0,940 & 0,964 & 0,914 \\
\hline sd & 0,059 & 0,051 & 0,046 & 0,041 & 0,285 & 0,042 & 0,036 & 0,036 & 0,056 \\
\hline Teste $\mathrm{t}$ & $\mathrm{S}$ & $\mathrm{S}$ & $\mathrm{S}$ & $\mathrm{S}$ & $\mathrm{Ns}$ & $\mathrm{S}$ & $\mathrm{ns}$ & ns & $\mathrm{ns}$ \\
\hline $\mathrm{R}^{2}$ & 94,40 & 94,81 & 96,21 & 98,08 & 33,13 & 97,87 & 99,00 & 98,92 & 97,05 \\
\hline \multicolumn{10}{|l|}{ Fêmeas } \\
\hline \multicolumn{10}{|l|}{ Females } \\
\hline A & $-0,683$ & $-0,822$ & $-0,824$ & $-0,830$ & $-0,923$ & $-0,612$ & $-1,613$ & $-1,659$ & $-1,548$ \\
\hline $\mathrm{b}$ & 0,616 & 0,654 & 0,656 & 0,763 & 0,788 & 0,703 & 0,979 & 0,992 & 0,961 \\
\hline sd & 0,060 & 0,053 & 0,046 & 0,050 & 0,049 & 0,054 & 0,040 & 0,034 & 0,033 \\
\hline Teste $\mathrm{t}$ & $\mathrm{S}$ & $\mathrm{S}$ & $\mathrm{s}$ & $\mathrm{S}$ & $\mathrm{S}$ & $\mathrm{ns}$ & ns & $\mathrm{ns}$ & ns \\
\hline $\mathrm{R}^{2}$ & 93,81 & 94,93 & 96,21 & 97,04 & 96,94 & 95,43 & 98,84 & 99,06 & 99,05 \\
\hline
\end{tabular}

$\mathrm{M} 1$ = método um; M2 = método dois e M3 = método três.

${ }^{*} \mathrm{~A}=$ intercepto; * $\mathrm{b}=$ coeficiente alométrico; * $\mathrm{sd}=$ erro-padrão; $\mathrm{s}=$ significativo para $(\mathrm{P}<0,05)$; ns $=$ não-significativo para $(\mathrm{P}>0,05)$. $M 1=$ method $1 ; M 2=$ method $2 ; M 3=$ method $3 ; A=$ intercept $; b=$ allometric coefficient; $s d=$ standard error; $s=$ significant at $(P<.05) ; n s=$ not significant at $(P>$.05). 
peso vivo indicam que o coração e o pulmão + traquéia apresentam crescimento heterogônico negativo $(b<1)$, o que caracteriza um desenvolvimento precoce em relação ao peso do corpo vazio. De acordo com Berg \& Butterfield (1976), os órgãos de maior importância vital têm maior crescimento em fase mais precoce da vida do animal.

Os resultados do presente trabalho corroboram os de Tovar et al. (1986), citado por Roque (1998), os quais verificaram que o coração e os pulmões são órgãos de crescimento precoce, diminuindo suas proporções com elevação do peso e decaindo progressivamente com o crescimento do animal. Quanto ao fígado os resultados são semelhantes aos de Silva et al. (1999), que encontraram coeficiente de crescimento de 0,927 para animais do mesmo grupo genético utilizado neste estudo.

Benévent (1971), ao estimar coeficientes de alometria de órgãos e tecidos de cordeiros machos e fêmeas da raça Merinos D'Árles, concluiu que os órgãos precoces no período pré-natal têm um desenvolvimento mais lento no período pós-natal. Para Kirton et al. (1972), a maioria dos órgãos internos dos ovinos são de maturidade precoce. O cérebro representa o extremo, atingindo $90 \%$ de seu tamanho máximo no momento em que o animal tem $35 \%$ do peso adulto, com crescimento menor conforme o animal vai atingindo a sua maturidade.

$\mathrm{Na}$ Tabela 4 são apresentados os valores percentuais de rúmen + retículo, aparelho digestivo (rúmen + retículo, abomaso, omaso, intestino delgado e intestino grosso), conteúdo digestivo e gordura interna em relação ao peso de corpo vazio (PCV). Os resultados mostram que entre métodos de alimentação houve diferença entre M2 e M3 para rúmen mais retículo e que o trato digestivo teve maior proporção no M2 em relação a M1 e M3 os quais não diferem entre si. Esta diferença do M2 em relação ao M3 para rúmen + retículo explica-se em função dos cordeiros do M2 terem sidos desmamados 15 dias antes, o que forçou a consumirem maior quantidade de alimento sólido, ocasionando desenvolvimento do rúmen mais precocemente. Estes resultados são concordantes com Lyford (1993), o qual afirma que o crescimento do pré-estômago é maior em animais que consomem alimentos sólidos nas primeiras semanas de vida. Para o mesmo autor, todos os órgãos do tubo digestivo, com exceção do intestino delgado, aumentam de peso em seus tecidos desde o momento de diferenciação no embrião até o animal adulto.

Para gordura interna, houve diferença significativa $(\mathrm{P}<0,05)$ entre métodos, sendo que os animais do M2 tiveram menor proporção em relação aos demais. Este fato está relacionado ao menor peso ao desmame destes animais como explicado anteriormente. Entre sexos as fêmeas tiveram maior deposição em relação aos machos, o que se deve a maior precocidade das mesmas.(Sierra, 1973).

Quanto aos componentes do peso vivo a variação de cada órgão, em importância relativa, ao peso do corpo vazio (PVC) foi diferente em cada caso. Alguns órgãos incrementaram sua proporção dentro do organismo $(\mathrm{P}<0,05)$, e outros não variaram, entre métodos, sexo e peso de abate, demostrando que alguns órgãos perdem sua importância dentro do conjunto como é o caso da cabeça e patas à medida

Tabela 4 - Proporções médias do componentes do peso vivo em relação ao peso de corpo vazio em três métodos de alimentação (M1, M2, M3), de cordeiros machos (M) e fêmeas (F) e pesos de abate de 25 (P1) e $33 \mathrm{~kg}$ (P2) de peso vivo

Table 4 - Average proportions of components of live weight in relation to the empty body weight in three feeding methods (M1, M2, M3) of male (M) and female (F) lambs; weights of slaughter at $25 \mathrm{~kg}(P 1)$ and $33 \mathrm{~kg}(P 2)$ of live body weight

\begin{tabular}{|c|c|c|c|c|c|c|c|}
\hline $\begin{array}{l}\text { Componentes } \\
\text { Components }\end{array}$ & M1 & M2 & M3 & $\mathrm{M}$ & $\mathrm{F}$ & P1 & $\mathrm{P} 2$ \\
\hline $\begin{array}{l}\text { Rúmen + retículo } \\
\text { Rumen + small net }\end{array}$ & $2,303^{\mathrm{ab}}$ & $2,419^{\mathrm{a}}$ & $2,138^{b}$ & 2,329 & 2,245 & 2,295 & 2,279 \\
\hline $\begin{array}{l}\text { Trato digestivo } \\
\text { Digestive tract }\end{array}$ & $7,407^{\mathrm{b}}$ & $8,700^{\mathrm{a}}$ & $7,304^{\mathrm{b}}$ & 8,055 & 7,552 & 8,049 & 7,558 \\
\hline $\begin{array}{l}\text { Gordura interna } \\
\text { Internal fat }\end{array}$ & $2,430^{\mathrm{b}}$ & $1,915^{\mathrm{a}}$ & $2,514^{\mathrm{a}}$ & $2,027^{\mathrm{b}}$ & $2,546^{\mathrm{a}}$ & 2,133 & 2,439 \\
\hline
\end{tabular}

Médias seguidas na linha para (M1, M2 e M3) (M e F) (P1 e P2), com letras desiguais são significativamente diferentes pelo teste Pdiff $(\mathrm{P}<0,05)$.

Means within a row (M1, M2 and M3) (M and F) (P1 e $P 2)$ followed by different letters are significantly different by $P$ diff test (P<.05).

R. Bras. Zootec., v.31, n.6, p.2290-2298, 2002 
Tabela 5 - Parâmetros das equações de regressão ( $\mathrm{A}$ e b) do logaritmo do peso dos rins, gordura perirenal e gordura interna, em função do logaritmo do peso de corpo vazio, erro-padrão, teste t $(H o: b=1)$ e coeficientes de determinação para sexo e métodos de alimentação

Table 5 - Parameters of regression equations $(A$ and $b)$ of the logarithmic of weight of kidneys, kidney fat and internal fat in relation to the logarithmic of the empty body weight, standard error, test $t(H 0: b=1)$ and coefficients of determination to sex and feeding methods

\begin{tabular}{|c|c|c|c|c|c|c|c|c|c|}
\hline & \multicolumn{3}{|c|}{$\begin{array}{c}\text { Rins } \\
\text { Kidneys }\end{array}$} & \multicolumn{3}{|c|}{$\begin{array}{c}\text { Gordura perirenal } \\
\text { Kidneyfat }\end{array}$} & \multicolumn{3}{|c|}{$\begin{array}{c}\text { Gordura interna } \\
\text { Internal fat }\end{array}$} \\
\hline & M1 & M2 & M3 & M1 & M2 & M3 & M1 & M2 & M3 \\
\hline \multicolumn{10}{|l|}{ Machos } \\
\hline A & $-0,996$ & $-1,150$ & $-1,107$ & $-1,611$ & $-1,241$ & $-1,885$ & $-3,149$ & $-2,374$ & $-2,751$ \\
\hline $\mathrm{b}$ & 0,670 & 0,712 & 0,700 & 0,869 & 0,760 & 0,946 & 1,350 & 1,130 & 1,239 \\
\hline sd & 0,027 & 0,029 & 0,028 & 0,108 & 0,160 & 0,121 & 0,081 & 0,95 & 0,133 \\
\hline Teste $\mathrm{t}$ & $\mathrm{S}$ & $\mathrm{S}$ & $\mathrm{S}$ & $\mathrm{ns}$ & $\mathrm{ns}$ & $\mathrm{ns}$ & S & $\mathrm{ns}$ & $\mathrm{ns}$ \\
\hline $\mathrm{R}^{2}$ & 98,86 & 98,73 & 98,77 & 90,16 & 73,78 & 88,50 & 97,88 & 95,30 & 92,59 \\
\hline \multicolumn{10}{|l|}{ Fêmeas } \\
\hline Females & & & & & & & & & \\
\hline A & $-0,936$ & $-1,138$ & $-0,826$ & $-2,189$ & $-1,759$ & $-2,570$ & $-2,672$ & $-2,603$ & $-3,118$ \\
\hline b & 0,646 & 0,702 & 0,617 & 0,760 & 0,913 & 1,137 & 1,241 & 1,216 & 1,360 \\
\hline sd & 0,021 & 0,034 & 0,052 & 0,100 & 0,059 & 0,100 & 0,041 & 0,149 & 0,139 \\
\hline Teste $\mathrm{t}$ & $\mathrm{s}$ & $\mathrm{S}$ & $\mathrm{S}$ & $\mathrm{ns}$ & $\mathrm{ns}$ & $\mathrm{ns}$ & $\mathrm{S}$ & $\mathrm{S}$ & $\mathrm{S}$ \\
\hline $\mathrm{R}^{2}$ & 99,24 & 98,15 & 94,65 & 93,75 & 96,73 & 94,18 & 99,57 & 93,03 & 95,02 \\
\hline
\end{tabular}

$\mathrm{M} 1$ = método um; $\mathrm{M} 2$ = método dois; $\mathrm{M} 3=$ método três; * $\mathrm{G}$ renal $=$ Gordura perirenal; ${ }^{*} \mathrm{G}$ interna = gordura interna; $\mathrm{s}=$ significativo para $(P<0,05) ;$ ns $=$ não-significativo para $(P>0,05)$.

$M 1=$ method $1 ; M 2=$ method $2 ; M 3=$ method $3 ; A=$ intercept $; b=$ allometric coefficient; $s d=$ standard error; $s=$ significant at $(P<.05), n s=$ not significant at $(P>05)$.

que aumenta o peso de sacrifício.

Na Tabela 5, são apresentados os coeficientes de alometria para rins, gordura perirenal e gordura interna, obtidos em função do peso de corpo vazio (PVC).

Os coeficientes de alometria mostram que os rins, independentemente de métodos de alimentação, são de crescimento precoce $(b<1)$ com desenvolvimento heterogônico negativo em relação ao todo, enquanto a gordura perirenal apresentou crescimento isométrico $(b=1)$.

O crescimento da gordura interna é isométrico $(b=1)$ no M2 e M3 apenas para machos. No entanto, para fêmeas, independentemente de métodos de alimentação, o crescimento é tardio ( $b>1$ ). Os coeficientes alométricos obtido para os rins, independentemente de sexo e métodos de alimentação, estão próximos dos encontrados por Osório et al. (1994), que, ao trabalharem com as raças Ideal e Texel, verificaram coeficiente de alometria de 0,753 e 0,751 , respectivamente. Os mesmos autores verificaram para gordura pélvica e gordura renal coeficientes de 2,358 e 3,576, respectivamente. Estes valores estão acima dos encontrados para a gordura perirenal do presente trabalho.

Os resultados encontrados para a gordura interna, nas fêmeas e machos do M1 são corroborados por Berg \& Walters (1983) os quais consideram que o crescimento da gordura começa relativamente devagar e aumenta geometricamente quando os animais entram na fase de acabamento.

Na Tabela 6, são apresentados os coeficientes de alometria do trato digestivo (rúmen + retículo, omaso, abomaso, intestino delgado e grosso) e rúmen + retículo obtidos em função do peso de corpo vazio (PCV).

Verifica-se, de modo geral, que o trato digestivo apresentou crescimento heterogônico tardio (b>1), exceto nas fêmeas do M1 e M3 (b=1). O rúmen + retículo é também de crescimento tardio (b>1). Os resultados mostram que, quanto maior o tempo para terminação dos cordeiros, mais desenvolvido será o rúmen- retículo e em consequência sua contribuição relativa ao peso vivo do animal será cada vez maior, o que contribuirá para redução do rendimento da carcaça.

Os resultados encontrados nesta pesquisa em relação ao trato digestivo são concordantes com os 
Tabela 6 -Parâmetros das equações de regressão ( $A$ e b) do logaritmo do peso do aparelho digestivo e rúmen + retículo, em função do logaritmo do peso de corpo vazio, erro padrão, teste t $(H o: b=1)$ e coeficientes de determinação para sexo e métodos de alimentação

Table 6 - Parameters of the regression equations (A and B) of the logarithmic of the weight of digestive tract and rumen + small net in relation to the empty body weight, standard error, test $t\left(H_{0}: b=1\right)$ and coefficients of determination to genre and feeding methods

\begin{tabular}{|c|c|c|c|c|c|c|}
\hline & \multicolumn{3}{|c|}{$\begin{array}{c}\text { Aparelho digestivo } \\
\text { Digestive tract }\end{array}$} & \multicolumn{3}{|c|}{$\begin{array}{l}\text { Rúmen + retículo } \\
\text { Rumem+ small net }\end{array}$} \\
\hline & M1 & M2 & M3 & M1 & M2 & M3 \\
\hline \multicolumn{7}{|l|}{ Machos } \\
\hline \multicolumn{7}{|l|}{ Males } \\
\hline A & $-1,992$ & $-2,441$ & $-2,130$ & $-7,652$ & $-8,018$ & $-7,608$ \\
\hline $\mathrm{b}$ & 1,195 & 1,318 & 1,230 & 2,369 & 2,468 & 2,351 \\
\hline sd & 0,055 & 0,0702 & 0,58 & 0,159 & 0,141 & 0,126 \\
\hline Teste $\mathrm{t}$ & $\mathrm{S}$ & $\mathrm{S}$ & $\mathrm{S}$ & $\mathrm{S}$ & $\mathrm{S}$ & $\mathrm{S}$ \\
\hline $\mathrm{R}^{2}$ & 98,55 & 97,78 & 98,23 & 97,36 & 97,78 & 98,02 \\
\hline \multicolumn{7}{|l|}{ Fêmeas } \\
\hline \multicolumn{7}{|l|}{ Females } \\
\hline A & $-1,722$ & $-1,946$ & $-1,537$ & $-7,136$ & $-7,151$ & $-7,039$ \\
\hline B & 1,134 & 1,197 & 1,084 & 2,255 & 2,258 & 2,223 \\
\hline $\mathrm{Sd}$ & 0,067 & 0,038 & 0,055 & 0,199 & 0,132 & 0,116 \\
\hline Teste $\mathrm{t}$ & $\mathrm{ns}$ & $\mathrm{S}$ & $\mathrm{ns}$ & $\mathrm{S}$ & $\mathrm{S}$ & $\mathrm{S}$ \\
\hline $\mathrm{R}^{2}$ & 97,63 & 99,19 & 98,23 & 96,98 & 98,31 & 98,66 \\
\hline
\end{tabular}

M1 = método um; M2 = método dois; M3 = método três.

${ }^{*} A=$ intercepto; * $b=$ coeficiente alométrico; * $s d=$ erro-padrão; $s=$ significativo para $(P<0,05)$; ns = não-significativo para $(P>0,05)$.

$M 1=$ method 1; M2 = method 2; $M 3=$ method $3 ; A=$ intercept $b=$ allometric coefficient; $s d=$ standard error; $s=$ significant $(P<.05)$, $n s=$ not significant at $(P>.05)$.

de Silva et al.(1999), que verificou coeficientes de crescimento de 1,705 para trato digestivo total, caracterizando um crescimento heterogônico tardio. Para rúmen + retículo os resultados estão próximos aos encontrados por Huidobro \& Villapadierna (1992), que observaram coeficientes de crescimento para pré-estômago de 2,976.

\section{Conclusões}

A cabeça, patas, rins e pulmão mais traquéia exercem maior influência sobre o peso vivo dos ovinos jovens, uma vez que suas proporções diminuem com a elevação do peso de abate de 25 para $33 \mathrm{~kg}$, o inverso ocorre com a gordura perirenal.

Em relação ao peso de corpo vazio, o crescimento das patas, coração, pulmão + traquéia e rins é precoce e o da pele, do fígado e da gordura perirenal, isométrico.

O trato gastrintestinal dos machos em relação ao corpo vazio cresce mais tardiamente que o das fêmeas; para a gordura interna a situação é inversa.

O crescimento do rúmen e retículo é tardio, independentemente de método de alimentação e sexo.

\section{Literatura Citada}

BENÉVENTT, M. Croissance relative ponderále postnatale, dans les deux sexes, des principaux tissus et arganes de lagneau Mérinos d'Arles. Annals de Biologie Animale, Biochimie et Biophysique, v.11, n.1, p.5-39, 1971.

BERG, R.T.; BUTTERFIELD, R.M. New concepts of cattle growth. Sydney: Sydney University Press, 1976. 240p.

BERG, R.T.; WALTERS, L.E. The meat animal changes and challenges. Journal of Animal Science, v.57, n.2, p.135146,1983

BLACK, J.L. Crecimento y desarrollo de corderos. In: HARESING, W. (Ed.) Producción ovina. México: AGT Editor, 1989. 592p.

BUTTERFIELD, R.M. New concepts of sheep growth. Sydney: University of Sydney, 1988. 168p.

COLOMER-ROCHER, F. Tabla para classificación de canales ovinas. Hoja Técnica INIA n.3, Madrid, 1974.

DELFA, R.; GONZALEZ, C.; TEIXEIRA, A. El quinto quarto, v.17, p.49-66, 1991.

DROUILLARD, J.S.; KLOPFENSTEIN, T.J.; BRITTON, R.A. et al. Growth, body composition, and visceral organ mass and metabolism in lambs during and after metabolizable protein or net energy restrictions. Journal of Animal Science, v.69, p.3357-3375, 1991.

HUIDOBRO, R., CAÑEQUE, V. Producción de carne de corderos de raza Manchega. 5. Crecimiento relativo del quinto cuarto y de los tejidos y piezas de la canal. Investigacion Agraria: Producción y Sanidad Animals, n.2, v.9, p.95-108, 1994. 
HUIDOBRO, F.R.; VILLAPADIERNA, A. Estudios sobre crescimento y desarrolo en corderos de raza Manchega. Madrid, 1992. 191p. (Tesis Doctoral) - Facultad de Veterinaria, Universidad Complutense, 1992.

HUXLEY, J.S. Problems of relative growth. London: Methuen, 1932. 276p.

JENKINS, T.G.; LEYMASTER, K.A. Estimates of maturing rates and masses at maturity for body components of sheep. Journal of Animal Science, v.71, p.2952-2957, 1993.

KIRTON, A.H.; FOURIE, P.D.; JURY, K.E. Growth and development of sheep. 3. Growth of carcass and non-carcass components of the southdown and Rommney and thir cross and some relationships with composition. New Zealand Journal of Agricultural Research, v.15, p.214- 217, 1972.

LYFORD, S.J. El rumiante fisiología digestiva y nutrición. Crescimento y desarrolho del aparato digestivo de los ruminantes. Zaragosa: Acribia, 1993. p.47-68.

NATIONAL RESEARCH COUNCIL - NRC. Nutrient requirements of sheep. 6.ed. Washington, D.C.: National Academy Press, 1985. 99p.

OLIVER, W.M.; CARPENTER, Z.C.; KING, G.T. et al. Qualitative and quantitative characteristics of ram, whether and ewe lamb carcasses. Journal of Animal Science, v.26, p.307, 1967

(Citar página)OSÓRIO, J.C.S.; GUERREIRO, J.L.V. Produção de carne na raça Ideal. In: ENCONTRO DE CRIADORES DE RAÇA IDEAL, 1., 1994, Pelotas. Resumo... Pelotas: Universidade Federal de Pelotas, 1994.

(Citar local e editora)PEYRON, C. La qualité de l'agneau de lou cherie. $1963.101 \mathrm{p}$.
ROQUE, A. P. Desenvolvimento relativo dos componentes do peso vivo, da composição regional e tecidual em cordeiros de cinco raças. Pelotas: Universidade Federal de Pelotas, 1998. 70p. Dissertação (Mestrado em Zootecnia) Universidade Federal de Pelotas, 1998.

ROQUE, A.P.; OSÓRIO, J.C.S.; FARIA, H.V. et al. Desenvolvimento relativo dos componentes do peso vivo, da composição regional e tecidual em cordeiros Corriedale. In: REUNIÃO ANUAL DA SOCIEDADE BRASILEIRA DE ZOOTECNIA, 34., 1997, Juiz de Fora. Anais... Juiz de Fora: Sociedade Brasileira de Zootecnia, 1997. p.296-298.

SAS INSTITUTE. SAS User's guide: statistics. v.2, version 6, 4.ed., Cary: 1996. 300p.

SIERRA, I. Producción de cordero jóven y pesado en la raza Rosa Aragonesa. I.E.P.G.E., n.18, 28p, 1973.

SILVA, L.F.; PIRES, C.C.; GUERRA, D.P. et al. Crescimento de osso, músculo, gordura e principais cortes da carcaça de cordeiros abatidos com diferentes pesos. In: REUNIÃO ANUAL DA SOCIEDADE BRASILEIRA DE ZOOTECNIA, 36., 1999, Porto Alegre. Anais...Porto Alegre: Sociedade Brasileira de Zootecnia, 1999. p.353.

Recebido em: 27/11/01 Aceito em: 09/07/02 